\title{
Urban Air Pollution and Emergency Department Visits for Cardiac and Respiratory Diseases
}

Mieczyslaw Szyszkowicz ${ }^{1,}{ }^{*}$, Nicholas de Angelis ${ }^{2}$

1. Health Canada, 251 Sir Frederick Banting Driveway, Ottawa, Canada; E-Mail: mietek.szyszkowicz@hc-sc.gc.ca

2. Carleton University, 1125 Colonel By Drive, Ottawa, Canada; E-Mail: nickdeangelis@cmail.carleton.ca

* Correspondence: Mieczyslaw Szyszkowicz; E-Mail: mietek.szyszkowicz@hc-sc.gc.ca

Academic Editor: Raghava R. Kommalapati

Special Issue: Air Pollution and Health

Adv Environ Eng Res

2022, volume 3, issue 1

doi:10.21926/aeer.2201005
Received: September 18, 2021

Accepted: January 30, 2022

Published: February 11, 2022

\begin{abstract}
Air pollution affects various aspects of human health. Here, the associations between the number of emergency department visits for circulatory and respiratory problems and ambient air pollution in Toronto, Canada, in the period between April 2004 and December 2015 were studied. The health data were linked with urban air pollution data and weather factors. The conditional Poisson regression models were built for 18 strata (sex, age group, season), 8 exposure factors (air pollutants, indexes), and their 15 lags (0-14 days). Circulatory problems: the associations were intensified in the cold period (October - March) and were associated with the air quality health index (AQHI). The estimated relative risks for all patients in the cold period, for an increase of the AQHI by 1 , at lags 0,1 , and 2 were 1.017 and $95 \%$ confidence interval $(1.010,1.024), 1.014$ (1.007, 1.021), and 1.009 (1.002, 1.016). Respiratory problems: the analogous results for ozone and its increase by $12.8 \mathrm{ppb}$ at lags 3,4 , and 5 were 1.052 $(1.033,1.161), 1.039(1.020,1.121)$, and $1.027(1.008,1.082)$. It was observed that exposure to certain air pollutants (nitrogen dioxide, ozone, and the AQHI index) are associated with increased emergency department visits in both cardiac and respiratory health problems.
\end{abstract}

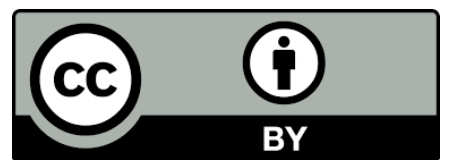

(C) 2022 by the author. This is an open access article distributed under the conditions of the Creative Commons by Attribution License, which permits unrestricted use, distribution, and reproduction in any medium or format, provided the original work is correctly cited. 


\section{Keywords}

Ambient air pollution; emergency; cardiac; health; respiratory

\section{Introduction}

The crowding of emergency departments (ED) has been shown to influence higher mortality rates, poorer patient outcomes or re-hospitalization and other detriments [1-3]. The Canadian Institute for Health Information $(\mathrm{ClHI})$ reports that the top three most common emergency department visits are for pulmonary and circulatory problems, specifically pneumonia, chronic obstructive pulmonary disease (COPD), and heart failure [4]. According to the Center for Disease Control and Prevention's National Hospital Ambulatory Medical Care Survey from 2017 (https://www.cdc.gov/nchs/data/nhamcs/web_tables/2017_ed_web_tables-508.pdf) diseases of respiratory and circulatory nature constituted $27.5 \%$ of ED visits in the United States. An improved understanding of ED visits may allow hospitals to improve diagnostic and treatment efficiency. A significant association between worse ambient air quality and ED visits, morbidity and mortality has been established [5].

The presented work proposes a more insightful method to analyse ED visits for cardiorespiratory health problems. The data analysed originate from a large urban area with intensive vehicle traffic. In this analysis we do not categorize health conditions, but rather analyse all cardiac and all respiratory health problems.

Here we test the hypothesis that ambient air pollution concentration level changes are associated with the number of ED visits related to cardiovascular and respiratory health conditions based on particular combinations of age group, sex, and seasons. The basis of the hypothesis is rooted in demonstrated impacts of certain air pollutants on circulatory and respiratory problems. Numerous studies have shown relationships between air pollutants and acute or chronic circulatory diseases via lung inflammation [6-8], blood borne mediators, neuroendocrine activation, or particle translocation [9]. The same can be said for respiratory diseases, where air pollution can be linked to higher risk for patients with asthma, COPD, lung cancer, or for those at increased risk of respiratory infection [10]. The PubMed search with the terms "air pollution cardiac" gives 3,824 publications and with "air pollution respiratory" gives 13,230 publications. The Integrated Science Assessments (EPA, https://www.epa.gov/isa) presents a potential biological pathway for each air pollutant considered in this work. This paper focuses on the short-term acute effects of air pollution, and selecting a time unit of one day.

\section{Materials and Methods}

We choose to group all diseases in the respective circulatory and respiratory categories (International Classification of Diseases, Tenth Revision - ICD-10 - codes 100-199 and J00-J99, respectively) as a collective, rather than investigate specific conditions. The goal of this paper is to investigate the correlations of the ED visit counts that are classified in these two categories with the variations in air pollution, using a large urban agglomeration in the city of Toronto, Canada, as the location of the study. 


\subsection{Health Data}

The National Ambulatory Care Reporting System (NACRS 2020) database was used to obtain data concerning the diagnosed emergency department visits in Toronto, Canada for the 4,292 days in the period between April 1st 2004 and December 31st 2015. The health cases where the primary cause of visit was categorized in the International Classification of Diseases: ICD-10 codes I00-I99 and J00-J99 (Chapter IX: "Diseases of the circulatory system", Chapter X: "Diseases of the respiratory system"), respectively, were extracted from the database. The health data were organized as daily counts of ED visits.

\subsection{Environmental Data}

Data on six ambient air pollutants were retrieved from the National Air Pollution Surveillance (NAPS) Program (managed by Environmental and Climate Change Canada) Canada-Wide Air Quality Database (CWAQD) for the same period. The pollutants considered separately are carbon monoxide (CO), nitrogen dioxide $\left(\mathrm{NO}_{2}\right)$, ground-level ozone $\left(\mathrm{O}_{3}\right.$, as a daily average, $\mathrm{O}_{3}$-h 8 , as a maximum eighthour average of ozone), fine particulate matter $\left(\mathrm{PM}_{2.5}\right)$, and sulfur dioxide $\left(\mathrm{SO}_{2}\right)$. These datasets have been used in previous epidemiological multi-sites study [11].

To account for multipollutant exposures, two air quality indexes ( $A Q H I$ and $A Q H I X)$ were calculated (see Table 1). The Air Quality Health Index (AQHI) is determined by the following formula, developed by Stieb et al. [12].

$$
A Q H I=\frac{1000}{10.4} \times\left(e^{0.000537 * 03}+e^{0.000871 * N O 2}+e^{0.000487 * P M 2.5}-3\right) .
$$

Table 1 summarizes statistics on air pollutants, temperature, and relative humidity in Toronto.

Table 1 Statistics on environmental factors (in 4,292 days). Toronto, Canada, 2004 - 2015.

\begin{tabular}{llllllll}
\hline Factors & Units & Min & Q1 & Median & Mean & Q3 & Max \\
\hline $\mathrm{PM} 25^{25}$ & $\mathrm{~g} / \mathrm{m}^{3}$ & 0.1 & 4.7 & 7.1 & 8.9 & 11.2 & 65.5 \\
$\mathrm{NO}_{2}$ & $\mathrm{ppb}$ & 3.2 & 11.1 & 15.0 & 16.1 & 19.9 & 59.8 \\
$\mathrm{O}_{3}$ & $\mathrm{ppb}$ & 1.7 & 16.8 & 23.0 & 23.5 & 29.6 & 62.1 \\
$\mathrm{O}_{3} \mathrm{H} 8$ & $\mathrm{ppb}$ & 9.0 & 33.0 & 41.0 & 43.7 & 52.0 & 107.0 \\
$\mathrm{SO}_{2}$ & $\mathrm{ppb}$ & 0.0 & 0.5 & 1.0 & 1.4 & 1.7 & 12.0 \\
$\mathrm{CO}$ & $\mathrm{ppm}$ & 0.0 & 0.2 & 0.2 & 0.3 & 0.3 & 1.1 \\
$\mathrm{AQHI}$ & number & 1.0 & 2.4 & 2.9 & 3.0 & 3.4 & 7.6 \\
$\mathrm{AQHIX}$ & number & 1.6 & 3.6 & 4.2 & 4.4 & 5.1 & 10.3 \\
Temperature & ${ }^{\circ} \mathrm{C}$ & -22.2 & 1.7 & 10.0 & 9.5 & 18.4 & 31.2 \\
Relative Humidity & $\%$ & 31.7 & 63.9 & 70.9 & 70.7 & 78.2 & 98.8 \\
\hline
\end{tabular}

Notes. Min - minimum, Max -maximum, Q1-25 th percentile, Q3-75 ${ }^{\text {th }}$ percentile.

The AQHI allows the estimate of risk associated with the ambient air quality to the general public and persons at risk using the three most prevalent pollutants $\left(\mathrm{O}_{3}, \mathrm{NO}_{2}\right.$, and $\left.\mathrm{PM}_{2.5}\right)$. While it is typically rounded to the nearest integer $(1-10,10+)$ in this study no rounding was performed for analysis. The AQHIX is used to account for the combination of $\mathrm{O}_{3} \mathrm{H} 8$ (an eight-hour average of $\mathrm{O}_{3}$ ), 
$\mathrm{NO}_{2}$ and $\mathrm{PM}_{2.5}$, calculated using the same formula. This index permits us to suppress the impact of $\mathrm{O}_{3}$ concentrations on $\mathrm{AQHI}$ associations.

\subsection{Statistical Approach}

Given the nature of the available data, a time stratified case-crossover technique was deemed most appropriate, as it controls for the time-invariant factors characteristic of each individual case. These confounders can be comorbidities, smoking, socioeconomic position, employment situation, and others. To eliminate the bias resulting from time-trends, the time-stratified design was realized and thus all similar weekdays within one month were grouped together. This methodology also allows the separation of strata such as "male" or "female" by seasonality, here identified as "cold" (October-March) and "warm" (April-September), to highlight the elevated risk to health when air pollutant effects are exacerbated by temperature and humidity. Temperature and relative humidity was incorporated into the model in the form of natural splines with three degrees of freedom. The generalized non-linear model procedure (the gnm package) with the "quasipoisson" option in $R$ statistical software (v. 4.0; 2020, The R Foundation for Statistical Computing) is used and estimates the coefficients and their standard errors for the considered air pollutants [13-16]. In this work, 2,160 statistical models are constructed for respiratory and cardiac health conditions. We applied the following statistical models

modelED <- gnm(EDCounts AirPL + ns(TempL,3) + ns(RHumL,3), data=dataEDvisits, family=quasipoisson, eliminate=factor(Cluster)).

The quasi-Poisson regression is applied to model an overdispersed count variable. Here air pollutant (AirPL), weather factors (temperature and relative humidity, TempL, RHumL), are lagged by the same number of days $(L)$. Here the lagged exposure by $L$ days means that the values of air pollutants and weather factors are used from previous $L$ days. The hierarchical Cluster has the form <year:month:day of week>. Such cluster contains 4 or 5 days. There are 1008 of such hierarchical levels (2004:1:1, ..., 2015:12:7). This structure of the clusters allows to control for time and its impact. Relative risk is calculated using the estimated coefficient for AirPL. The gnm package realizes Generalized Non-Linear Models technique.

The relative risk (RR) was calculated for each stratum at every lag and every pollutant measure, but to quantify the strength of the most significant associations, only the strongest relative risks are shown. The RR values with their $95 \%$ confidence intervals are expressed per interquartile range. All these values are listed in supplementary materials. A p-value less than 0.05 was considered statistically significant. The Health Canada Research Ethic Board determined that the study is IRB exempt, given that patient data were pre-existing and de-identified.

\section{Results}

In the period of the study, a total of 484,967 cases categorized in ICD codes 100-199 (diseases of the circulatory system) and 852,624 cases in ICD codes J00-J99 (diseases of the respiratory system) were recorded. 18 strata were used to separate cases by sex, age group and season. This allows identification of subgroups most affected by air pollution exposures.

Table 2 and Table 3 summarize the case count for each stratum in the circulatory disease categories and respiratory, respectively. Classified by sex were 235,301 male and 249,666 female circulatory disease cases over the same period. Classified by sex were 421,903 female and 430,721 
male respiratory cases in the studied period. It should be noted that in the case of kids (patients younger than 11 year of age, Table 2) for circulatory diseases the number of visits is very small. In this regard there may be a statistical power problem. Table 3 summarizes statistics on air pollutants, temperature, and relative humidity in Toronto.

Table 2 Statistics on emergency department (ED) visits for all cardiac problems (ICD-10 codes: 100-199). Toronto, Canada, April 2004 - December 2015.

\begin{tabular}{llllllll}
\hline Person/Age/Season & ED visits & Min & Q1 & Median & Mean & Q3 & Max \\
\hline All & 484,967 & 49 & 89 & 119 & 113.0 & 133 & 177 \\
Female & 235,301 & 19 & 45 & 56 & 54.8 & 64 & 96 \\
Male & 249,666 & 21 & 45 & 61 & 58.2 & 70 & 97 \\
Warm All & 246,331 & 50 & 88 & 118 & 112.2 & 132 & 174 \\
Warm Female & 119,641 & 19 & 45 & 56 & 54.5 & 64 & 96 \\
Warm Male & 126,690 & 21 & 45 & 61 & 57.7 & 69 & 93 \\
Cold All & 238,636 & 49 & 90 & 120 & 113.9 & 135 & 177 \\
Cold Female & 115,660 & 20 & 46 & 56 & 55.2 & 65 & 95 \\
Cold Male & 122,976 & 22 & 45 & 61 & 58.7 & 71 & 97 \\
Age 0-10 All & 1,764 & 0 & 0 & 1 & 0.9 & 1 & 6 \\
Age 0-10 Female & 1,286 & 0 & 0 & 0 & 0.3 & 0 & 4 \\
Age 0-10 Male & 1,990 & 0 & 0 & 0 & 0.5 & 1 & 5 \\
Age 11-60 All & 169,020 & 10 & 30 & 40 & 39.4 & 48 & 79 \\
Age 11-60 Female & 73,271 & 1 & 13 & 17 & 17.1 & 21 & 43 \\
Age 11-60 Male & 95,749 & 3 & 17 & 23 & 22.3 & 28 & 51 \\
Age 60+All & 312,671 & 27 & 59 & 75 & 72.9 & 86 & 120 \\
Age 60+ Female & 160,744 & 10 & 31 & 38 & 37.5 & 44 & 66 \\
Age 60+ Male & 151,927 & 10 & 28 & 36 & 35.4 & 43 & 65 \\
\hline
\end{tabular}

Notes. Min - minimum, Max -maximum, Q1-25 ${ }^{\text {th }}$ percentile, Q3-75 ${ }^{\text {th }}$ percentile.

Table 3 Statistics on ED visits for all respiratory problems (ICD-10 codes: J00-J99). Toronto, Canada, April 2004 - December 2015.

\begin{tabular}{llllllll}
\hline Person/Age/Season & ED visits & Min & Q1 & Median & Mean & Q3 & Max \\
\hline All & 852,624 & 86 & 161 & 188 & 198.7 & 220 & 751 \\
Female & 421,903 & 35 & 78 & 92 & 98.3 & 109 & 398 \\
Male & 430,721 & 39 & 82 & 96 & 100.4 & 113 & 358 \\
Warm All & 376,931 & 86 & 145 & 167 & 171.6 & 193 & 346 \\
Warm Female & 184,694 & 35 & 70 & 82 & 84.1 & 95 & 180 \\
Warm Male & 192,237 & 39 & 74 & 85 & 87.5 & 99 & 178 \\
Cold All & 475,693 & 125 & 185 & 209 & 227 & 244 & 751 \\
Cold Female & 237,209 & 53 & 90 & 103 & 113.2 & 123 & 398 \\
Cold Male & 238,484 & 51 & 94 & 106 & 113.8 & 124 & 358 \\
Age 0-10 All & 222,107 & 12 & 37 & 48 & 51.8 & 62 & 275 \\
Age 0-10 Female & 91,080 & 2 & 14 & 19 & 21.2 & 26 & 124
\end{tabular}




\begin{tabular}{llllllll} 
Age 0-10 Male & 131,027 & 4 & 22 & 29 & 30.5 & 37 & 162 \\
Age 11-60 All & 434,200 & 42 & 83 & 95 & 101.2 & 112 & 426 \\
Age 11-60 Female & 227,051 & 17 & 42 & 49 & 52.9 & 59 & 237 \\
Age 11-60 Male & 207,149 & 13 & 39 & 46 & 48.3 & 54 & 189 \\
Age 60+All & 196,317 & 14 & 37 & 43 & 45.7 & 51 & 188 \\
Age 60+ Female & 103,772 & 6 & 18 & 23 & 24.2 & 28 & 114 \\
Age 60+ Male & 92,545 & 5 & 17 & 21 & 21.6 & 25 & 76 \\
\hline
\end{tabular}

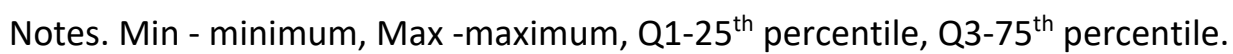

To show the associations between the ED visits by strata and air pollutants (lags), their positive and statistically significant correlations are presented in Figures 1, 2, 3, and 4. Other associations (negative and none) are shown as a zero. The significance is defined as a $p$-value $<0.05$. Using a $p$ value $<0.001$ rather than a $p$-value $<0.05$ as a criterion, 61 cases persist among 294 positive, and 420 among 919 positive, i.e., $20.7 \%$ and $45.5 \%$, for circulatory and respiratory ED visits, respectively.

Figure 1 shows all statistically significant positive correlations between pollution and ED visits considered by a stratum and a lag for a particular pollutant for both circulatory and respiratory cases. Positive correlations for respiratory cases are indicated with a 1, and circulatory cases with a 2 . A strata/lag combination showing 3 indicates an overlap of positive correlations for both respiratory and circulatory cases. If both are not positive, the cell contains a 0 . All associations, including negative, can be found in Supplementary Materials (Figure e1 (Cardiac) and Figure e2 (Respiratory)), as well as the corresponding values of numerical estimations of slope and associated standard error (files CARDIACToronto.csv, RESPIRATORYToronto.csv) at https://github.com/szyszkowiczm/CardioRespirTORONTO. The columns in these figures represent sequential lags (from 0 to 14 days). In all presented figures colors are used to ease visualization of association counts, where a darker red indicates a larger association count in terms of the criteria not featured on one of the table axes (either strata, lags, or pollutants). The corresponding figures are presented in the referenced Supplementary Materials. These materials also include histograms of the considered ambient air pollutants, temperature, and relative humidity. The map of the city of Toronto which shows population density and the locations of monitor stations is also provided. Two figures, Figure e3 (RR, Cardiac, $\mathrm{NO}_{2}$ ) and Figure e4 (RR, Respiratory, $\mathrm{O}_{3}$ ), which present a forest plot for lags from 0 to 3 for nitrogen dioxide and ozone are given. 


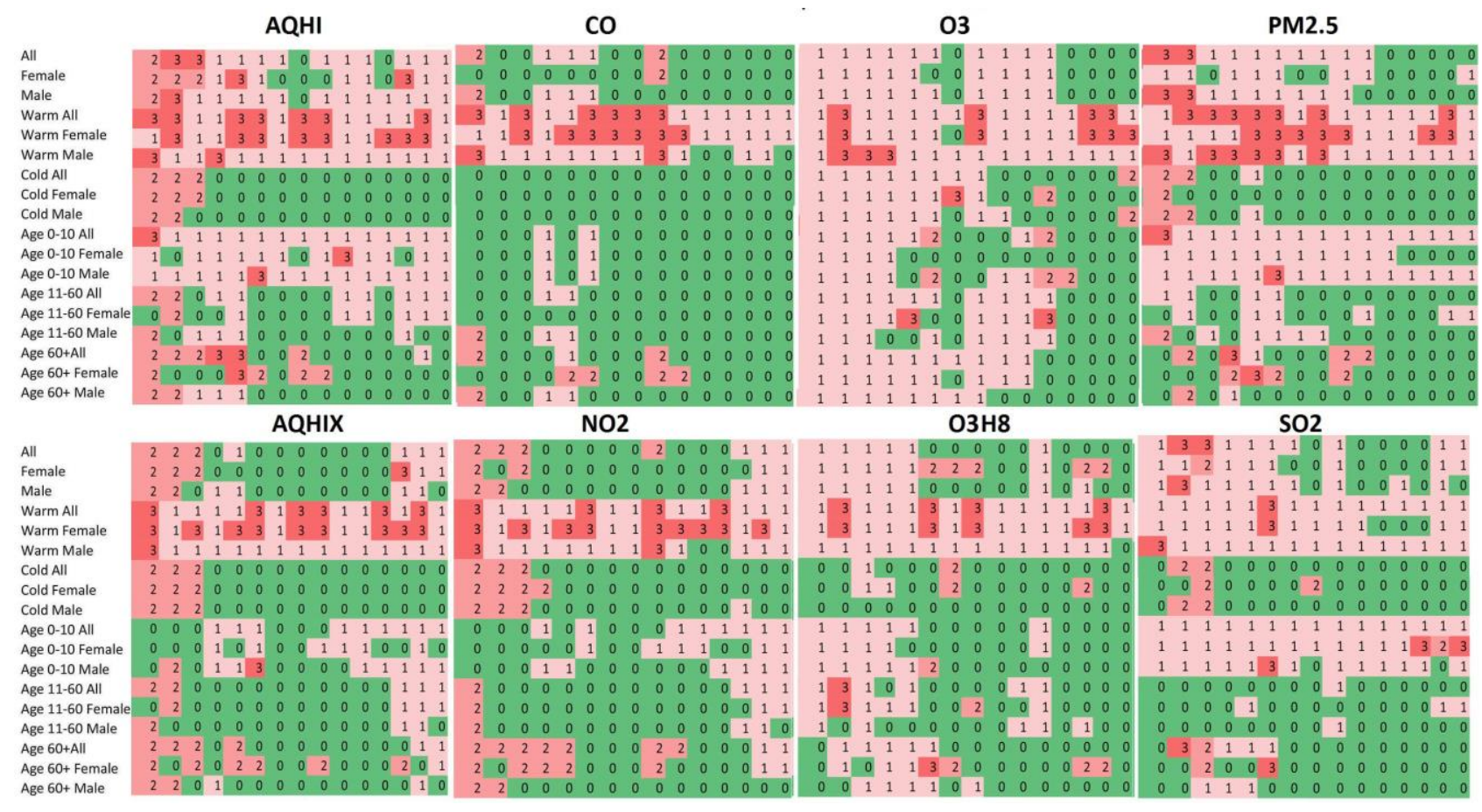

Figure 1 The associations between pollution and ED visits by a stratum, pollutant and lags (from 0 to 14 days) for ICD Codes I00-199 (Circulatory) indicated with a 2 and for ICD Codes J00-J99 (Respiratory) indicated with a 1. If both statistical significance associations overlap, a 3 is indicated. Other associations are shown by a 0.

Figure 2 illustrates a summary of both the circulatory (left) and respiratory (right) counts of models with statistically significant associations between pollution and ED visit considered for a particular stratum and every lag used (0 to 14). In other words, it shows the number of positive statistically significant associations between cardiovascular and respiratory ED visits and all air pollutants and $\mathrm{AQHI}$ combined.

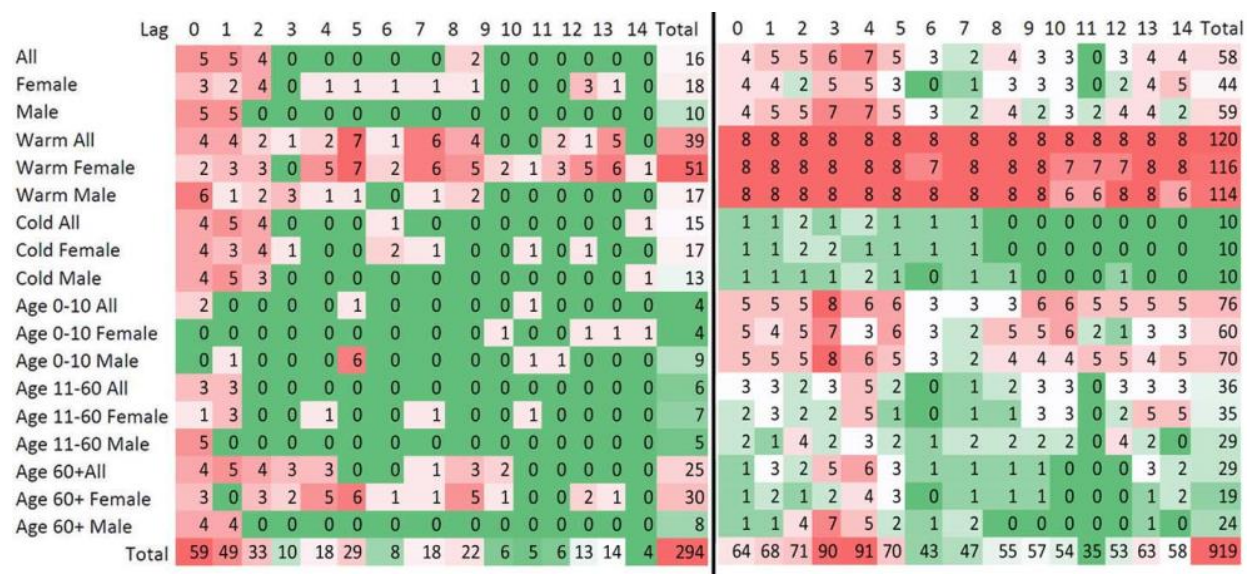

Figure 2 Total counts of positive statistically significant associations between pollution and ED visits by the considered stratum and lag (left: Circulatory, right: Respiratory).

Figure 3 plays a similar role, but it summarizes Figure 1 by statistically significant models between a pollutant and every lag considered. These two figures indicate that lags 0 and 1, lags 3 and 4 , saw the most positive significant association for circulatory and respiratory, respectively. The strata 
most affected are "cold all" and "cold female, "age 0-10 all" and "age 0-10 male" for circulatory and respiratory cases, respectively.

\begin{tabular}{|c|c|c|c|c|c|c|c|c|c|c|c|c|c|c|c|c|c|}
\hline \multirow{2}{*}{ Air Pollution: } & $A Q$ & $H I X$ & $\mathrm{rN}$ & 2 & \multicolumn{4}{|c|}{$\mathrm{O} 3 \mathrm{H} 8 \mathrm{SO} 2$} & \multicolumn{9}{|c|}{ AQHIX $\mathrm{NO} 2 \quad \mathrm{O} 3 \mathrm{H} 8 \quad \mathrm{SO} 2$} \\
\hline & AQHI & & $\mathrm{cO}$ & & O3 & PM2. & 5 To & tal & $\mathrm{AQH}$ & & $\mathrm{CO}$ & & 03 & & M2.5 & & Tot \\
\hline All & 3 & 3 & 2 & 4 & 00 & 02 & 2 & 16 & 12 & 4 & 3 & 3 & 10 & 6 & 10 & 10 & 58 \\
\hline Female & 5 & 4 & 1 & 2 & 05 & 0 & 1 & 18 & 8 & 3 & 0 & 2 & 9 & 6 & 8 & 8 & 44 \\
\hline Male & 2 & 2 & 1 & 2 & 00 & 2 & 1 & 10 & 13 & 4 & 3 & 3 & 10 & 7 & 9 & 10 & 59 \\
\hline larm All & 7 & 6 & 6 & 4 & 44 & 4 & 1 & 39 & 15 & 15 & 15 & 15 & 15 & 15 & 15 & 15 & 120 \\
\hline larm Fe & 8 & 9 & 7 & 9 & 55 & 7 & 1 & 51 & 15 & 15 & 15 & 15 & 14 & 15 & 15 & 12 & 116 \\
\hline & 2 & 1 & 2 & 2 & 30 & 6 & 1 & 17 & 15 & 15 & 12 & 13 & 15 & 14 & 15 & 15 & 114 \\
\hline & 3 & 3 & 0 & 3 & 11 & 2 & 2 & 15 & 0 & 0 & 0 & 0 & 8 & 1 & 1 & 0 & 10 \\
\hline Cold & 3 & 3 & 0 & 4 & 22 & 1 & 2 & 17 & 0 & 0 & 0 & 0 & 8 & 2 & 0 & 0 & 10 \\
\hline Cold & 2 & 3 & 0 & 3 & 10 & 2 & 2 & 13 & 0 & 0 & 0 & 1 & 8 & 0 & 1 & 0 & 10 \\
\hline Age 0-10 All & 1 & 0 & 0 & 0 & 20 & 1 & 0 & 4 & 15 & 9 & 2 & 8 & 6 & 6 & 15 & 15 & 76 \\
\hline ge $0-10$ & 1 & 0 & 0 & 0 & 00 & 0 & 3 & 4 & 12 & 6 & 2 & 6 & 4 & 5 & 11 & 14 & 60 \\
\hline Age 0-10 Male & 1 & 2 & 0 & 0 & 31 & 1 & 1 & 9 & 15 & 8 & 2 & 6 & 6 & 5 & 15 & 13 & 70 \\
\hline Age & 2 & 2 & 0 & 1 & 01 & 0 & 0 & 0 & 7 & 3 & 2 & 3 & 10 & 6 & 4 & 1 & 36 \\
\hline Age 11-60 Female & 1 & 1 & 0 & 1 & 22 & 0 & 0 & 7 & 6 & 3 & 0 & 2 & 9 & 6 & 6 & 3 & 35 \\
\hline Age 1 & 1 & 1 & 1 & 1 & 0 & 0 & 0 & 5 & 4 & 2 & 2 & 2 & 8 & 5 & 5 & 1 & 29 \\
\hline Age $60+$ All & 6 & 4 & 2 & 7 & 00 & 4 & 2 & 25 & 3 & 2 & 1 & 2 & 10 & 5 & 2 & 4 & 29 \\
\hline Age $60+$ Female & 5 & 6 & 4 & 5 & 04 & 4 & 2 & 30 & 1 & 1 & 0 & 2 & 9 & 4 & 1 & 1 & 19 \\
\hline Age $60+$ Male & 2 & 2 & 1 & 2 & 00 & $0 \quad 1$ & 0 & 8 & 3 & 2 & 2 & 0 & 8 & 5 & 1 & 3 & 24 \\
\hline Total & 555 & 52 & & & 2325 & 541 & 212 & 294 & 144 & 92 & 61 & 83 & 1671 & 113 & 134 & 125 & 919 \\
\hline
\end{tabular}

Figure 3 Total counts of statistically significant associations between pollution and ED visits by the considered stratum and pollutant or index (left: Circulatory, right: Respiratory).

Figure 4 summarizes the count of positive statistically significant associations between pollutants or indexes and ED visits for each considered lag. It shows that for circulatory cases, AQHI is a good metric of association as 55 out of the total 294 statistically significant positive associations between a pollutant or index and all strata were between $\mathrm{AQHI}$ and the lags, particularly in the first few lags (0-2). $\mathrm{NO}_{2}$ has the highest amount of associations of the mono-pollutant models. For respiratory cases, $\mathrm{O}_{3}$ is strongly associated with higher case frequency with a total of 167 associations. Similarly, to respiratory cases, the AQHI proves to be a good predictor of respiratory cases with a total of 144 associations.

\begin{tabular}{l|rrrrrrrrrrrrrrrr|rrrrrrrrrrrrrrrrrrrrr}
$\quad \mathrm{Lag}$ & 0 & 1 & 2 & 3 & 4 & 5 & 6 & 7 & 8 & 9 & 10 & 11 & 12 & 13 & 14 & Total & 0 & 1 & 2 & 3 & 4 & 5 & 6 & 7 & 8 & 9 & 10 & 11 & 12 & 13 & 14 & Total \\
$\mathrm{AQHI}$ & 14 & 12 & 5 & 2 & 5 & 4 & 0 & 4 & 3 & 1 & 0 & 1 & 2 & 2 & 0 & 55 & 6 & 7 & 10 & 13 & 15 & 9 & 8 & 5 & 8 & 11 & 11 & 7 & 11 & 12 & 11 & 144 \\
$\mathrm{AQHIX}$ & 14 & 11 & 8 & 0 & 3 & 4 & 0 & 2 & 3 & 0 & 0 & 2 & 3 & 2 & 0 & 52 & 3 & 3 & 3 & 8 & 7 & 6 & 3 & 3 & 4 & 5 & 6 & 5 & 11 & 14 & 11 & 92 \\
$\mathrm{CO}$ & 7 & 0 & 2 & 0 & 2 & 3 & 2 & 2 & 7 & 2 & 0 & 0 & 0 & 0 & 0 & 27 & 3 & 3 & 3 & 11 & 9 & 8 & 3 & 3 & 3 & 3 & 2 & 2 & 3 & 3 & 2 & 61 \\
$\mathrm{NO}_{2}$ & 15 & 7 & 8 & 3 & 3 & 2 & 0 & 0 & 6 & 2 & 1 & 2 & 0 & 1 & 0 & 50 & 3 & 3 & 3 & 5 & 4 & 5 & 3 & 3 & 4 & 5 & 4 & 4 & 10 & 14 & 13 & 83 \\
$\mathrm{O}_{3}$ & 0 & 3 & 1 & 1 & 1 & 2 & 1 & 2 & 0 & 0 & 4 & 1 & 2 & 2 & 3 & 23 & 18 & 18 & 18 & 17 & 15 & 13 & 6 & 15 & 13 & 13 & 9 & 3 & 3 & 3 & 3 & 167 \\
$\mathrm{O}_{3} \mathrm{H} 8$ & 0 & 4 & 0 & 0 & 0 & 5 & 4 & 4 & 0 & 0 & 0 & 0 & 4 & 4 & 0 & 25 & 12 & 13 & 16 & 14 & 13 & 6 & 3 & 4 & 3 & 5 & 11 & 3 & 5 & 3 & 2 & 113 \\
$\mathrm{PM}_{2.5}$ & 8 & 7 & 2 & 4 & 4 & 5 & 1 & 3 & 3 & 1 & 0 & 0 & 1 & 2 & 0 & 41 & 10 & 11 & 9 & 11 & 16 & 12 & 9 & 9 & 9 & 9 & 6 & 5 & 5 & 6 & 7 & 134 \\
$\mathrm{SO}_{2}$ & 1 & 5 & 7 & 0 & 0 & 4 & 0 & 1 & 0 & 0 & 0 & 0 & 1 & 1 & 1 & 21 & 9 & 10 & 9 & 11 & 12 & 11 & 8 & 5 & 11 & 6 & 5 & 6 & 5 & 8 & 9 & 125 \\
$\quad \mathrm{~T}$ tal & 59 & 49 & 33 & 10 & 18 & 29 & 8 & 18 & 22 & 6 & 5 & 6 & 13 & 14 & 4 & 294 & 64 & 68 & 71 & 90 & 91 & 70 & 43 & 47 & 55 & 57 & 54 & 35 & 53 & 63 & 58 & 919
\end{tabular}

Figure 4 Total counts of positive statistically significant associations between pollution and ED visits by the considered pollutant or index and lag (left: Circulatory, right: Respiratory).

The relative risk for lags $0,1,2$ (circulatory) and 3, 4, 5 (respiratory) for pollutants shown to be particularly correlative was calculated and shown in Tables 4 and 5. 
Table 4 Estimated RRs and their $95 \%$ confidence intervals (95\% Cl) for an increase of the $A Q H I$ value by a one interquartile range $(I Q R=1)$ for circulatory cases. Toronto, Canada, April 2004-December 2015.

\begin{tabular}{lllllll}
\hline Lags & \multicolumn{2}{c}{ Lag 0 } & \multicolumn{2}{l}{ Lag 1 } & \multicolumn{2}{l}{ Lag 2 } \\
\hline Person/Age/Season & RR & 95\%Cl & RR & 95\%Cl & RR & 95\%Cl \\
\hline All & 1.014 & $(1.008,1.021)$ & 1.011 & $(1.005,1.017)$ & 1.007 & $(1.001,1.013)$ \\
Female & 1.011 & $(1.003,1.019)$ & 1.011 & $(1.002,1.019)$ & 1.008 & $(1.000,1.016)$ \\
Male & 1.017 & $(1.009,1.026)$ & 1.011 & $(1.003,1.020)$ & 1.006 & $(0.998,1.014)$ \\
Warm All & 1.012 & $(1.006,1.018)$ & 1.009 & $(1.002,1.015)$ & 1.006 & $(1.000,1.012)$ \\
Warm Female & 1.007 & $(0.999,1.015)$ & 1.010 & $(1.002,1.018)$ & 1.006 & $(0.999,1.014)$ \\
Warm Male & 1.017 & $(1.009,1.025)$ & 1.008 & $(1.000,1.016)$ & 1.005 & $(0.997,1.013)$ \\
Cold All & 1.017 & $(1.010,1.024)$ & 1.014 & $(1.007,1.021)$ & 1.009 & $(1.002,1.016)$ \\
Cold Female & 1.016 & $(1.007,1.025)$ & 1.011 & $(1.002,1.020)$ & 1.010 & $(1.001,1.019)$ \\
Cold Male & 1.019 & $(1.010,1.027)$ & 1.017 & $(1.008,1.026)$ & 1.007 & $(0.998,1.016)$ \\
Age 0-10 All & 1.066 & $(1.003,1.133)$ & 1.019 & $(0.958,1.083)$ & 1.008 & $(0.947,1.073)$ \\
Age 0-10 Female & 1.065 & $(0.979,1.160)$ & 0.964 & $(0.883,1.051)$ & 1.052 & $(0.963,1.149)$ \\
Age 0-10 Male & 1.069 & $(0.993,1.150)$ & 1.053 & $(0.980,1.133)$ & 0.982 & $(0.911,1.059)$ \\
Age 11-60 All & 1.018 & $(1.008,1.028)$ & 1.012 & $(1.002,1.022)$ & 1.004 & $(0.994,1.014)$ \\
Age 11-60 Female & 1.012 & $(0.998,1.026)$ & 1.015 & $(1.001,1.030)$ & 1.009 & $(0.995,1.023)$ \\
Age 11-60 Male & 1.023 & $(1.010,1.035)$ & 1.010 & $(0.998,1.023)$ & 1.000 & $(0.988,1.012)$ \\
Age 60+All & 1.012 & $(1.004,1.019)$ & 1.010 & $(1.003,1.017)$ & 1.009 & $(1.001,1.016)$ \\
Age 60+ Female & 1.010 & $(1.001,1.019)$ & 1.009 & $(1.000,1.018)$ & 1.008 & $(0.998,1.017)$ \\
Age 60+ Male & 1.013 & $(1.004,1.023)$ & 1.012 & $(1.002,1.022)$ & 1.010 & $(1.000,1.020)$ \\
\hline
\end{tabular}

Table 5 Estimated RRs and their $95 \%$ confidence intervals (95\% Cl) Cls for an increase of concentration of ozone $\left(\mathrm{O}_{3}\right)$ by a one interquartile range ( $\left.\mathrm{IQR}=12.8 \mathrm{ppb}\right)$ for Respiratory cases. Toronto, Canada, April 2004-December 2015.

\begin{tabular}{|c|c|c|c|c|c|c|}
\hline Lags & & Lag 3 & & Lag 4 & & Lag 5 \\
\hline Person/Age/Season & $\mathbf{R} \mathbf{R}$ & $95 \% \mathrm{Cl}$ & $\mathbf{R R}$ & $95 \% \mathrm{Cl}$ & $\mathbf{R} \mathbf{R}$ & $95 \% \mathrm{Cl}$ \\
\hline All & 1.033 & $(1.019,1.101)$ & 1.025 & $(1.011,1.076)$ & 1.017 & $(1.003,1.052)$ \\
\hline Female & 1.042 & $(1.025,1.128)$ & 1.030 & $(1.014,1.093)$ & 1.016 & $(0.999,1.047)$ \\
\hline Male & 1.025 & $(1.010,1.076)$ & 1.020 & $(1.005,1.060)$ & 1.019 & $(1.004,1.057)$ \\
\hline Warm All & 1.019 & $(1.009,1.056)$ & 1.016 & $(1.007,1.049)$ & 1.014 & $(1.004,1.041)$ \\
\hline Warm Female & 1.020 & $(1.009,1.061)$ & 1.015 & $(1.004,1.046)$ & 1.013 & $(1.001,1.038)$ \\
\hline Warm Male & 1.017 & $(1.006,1.052)$ & 1.017 & $(1.006,1.052)$ & 1.014 & $(1.003,1.043)$ \\
\hline Cold All & 1.052 & $(1.033,1.161)$ & 1.039 & $(1.020,1.121)$ & 1.027 & $(1.008,1.082)$ \\
\hline Cold Female & 1.069 & $(1.047,1.217)$ & 1.051 & $(1.030,1.158)$ & 1.025 & $(1.004,1.075)$ \\
\hline Cold Male & 1.035 & $(1.016,1.107)$ & 1.028 & $(1.009,1.084)$ & 1.029 & $(1.011,1.090)$ \\
\hline Age 0-10 All & 1.035 & $(1.015,1.108)$ & 1.020 & $(1.000,1.062)$ & 1.005 & $(0.985,1.014)$ \\
\hline Age 0-10 Female & 1.045 & $(1.019,1.138)$ & 1.024 & $(0.999,1.073)$ & 0.989 & $(0.964,0.966)$ \\
\hline Age 0-10 Male & 1.029 & $(1.006,1.087)$ & 1.018 & $(0.996,1.054)$ & 1.016 & $(0.994,1.049)$ \\
\hline Age 11-60 All & 1.029 & $(1.012,1.087)$ & 1.020 & $(1.004,1.060)$ & 1.017 & $(1.001,1.051)$ \\
\hline
\end{tabular}




\begin{tabular}{lllllll} 
Age 11-60 Female & 1.040 & $(1.021,1.123)$ & 1.023 & $(1.004,1.068)$ & 1.015 & $(0.996,1.045)$ \\
Age 11-60 Male & 1.017 & $(0.999,1.050)$ & 1.017 & $(0.999,1.051)$ & 1.019 & $(1.001,1.057)$ \\
Age 60+All & 1.041 & $(1.023,1.125)$ & 1.042 & $(1.023,1.128)$ & 1.032 & $(1.014,1.099)$ \\
Age 60+ Female & 1.042 & $(1.019,1.130)$ & 1.053 & $(1.030,1.165)$ & 1.041 & $(1.018,1.126)$ \\
Age 60+ Male & 1.039 & $(1.017,1.120)$ & 1.029 & $(1.007,1.088)$ & 1.023 & $(1.002,1.070)$ \\
\hline
\end{tabular}

As mentioned earlier, for circulatory cases, the "cold all" and "cold female" strata were most affected at these lags. The relative risk for these strata at lags 0 and 1 was 1.017 and 1.012, 1.015 and 1.011, respectively. It is noted that the RR for ages 0 to 10 is very high, however this is due to the relatively small total case count. For respiratory cases, lags 3 and 4 were most significant, with "age 0-10 all" and "age 0-10 male" having the most associations among all strata. At lags 3 and 4, these strata had relative risks of 1.035 and $1.029,1.020$ and 1.018, respectively. The relative risks and their $95 \%$ confidence intervals were calculated for an increase of one IQR of air pollutant for all considered combinations of air pollutants, their lags, and strata. Their values are re-ported in the following files: CARDIACRRiskToronto.csv and RESPIRATORYRRiskToronto.csv, found in Supplementary Materials.

\section{Discussion}

Numerous studies have shown a strong correlation between elevated ambient air pollution levels and increased rates of mortality and morbidity associated with respiratory [17-19], and circulatory complications $[6,8,19,20]$. It was therefore expected to find that there were many positive associations between certain strata and air pollutants. For respiratory disease-related cases, it was observed that lags 3 and 4 were most significant, thus acute responses to short-term air pollution exposure are highly likely compared to other lags. This observation is reflected by previous findings, as it has been shown by D'amato et al. [17] that ambient air pollution can significantly in-crease the risk of respiratory illness and aggravate pre-existing conditions in the short-term. Our results also showed a significant correlation between individuals aged 0-10 years and visits to the ED. This association has been observed in many studies [10, 21, 22], which have highlighted increases in emergency department visits related to respiratory illness among children (aged 5-9 years) related to short-term exposure to air pollution [22]. Our results follow the findings of Alhanti et al. [23], that the highest for asthma-related (most common chronic respiratory illness in Canada according to Statistics Canada) ED visits concerned the 5 to 18 year age group. Our results showed that out of all individual pollutants, the highest count of positive associations for respiratory cases was found to be with $\mathrm{O}_{3}$. Ozone has been shown to be a significant factor in worsening of respiratory conditions [19], however we report few associations between $\mathrm{O}_{3}$ and the most affected strata, children 0-10. While ozone has elevated counts of positive associations with all other strata relatively to some other pollutants, the 0-10 age group irrespective of sex is uniquely affected by $\mathrm{PM}_{2.5}$ and $\mathrm{SO}_{2}$ levels. A possible explanation to this observation is that children spend less time out-side on hotter days, when $\mathrm{O}_{3}$ levels are elevated due to elevated temperature.

As with respiratory illnesses, air pollution is also known to have a detrimental effect on the circulatory system $[19,20]$. This is especially the case for particulate matter $\left(\mathrm{PM}_{2.5}\right)[19,24]$ and $\mathrm{NO}_{2}$, $[19,25]$ corresponding to the two highest mono-pollutant positive association counts in this study (34 and 45 respectively). ED visits related to circulatory problems saw positive associations at the 
shortest lags ( 0 and 1 days in particular), consistent with previous findings [19]. It has been shown that in early lags, exposure to $\mathrm{NO}_{2}$ can increase circulatory inflammation by triggering an elevated level of high-sensitivity C-reactive protein [26]. Our results also show that adverse circulatory response is aggravated in the cold months, a relationship which has been demonstrated previously [27]. In colder months, ambient air pollution levels are relatively high, contributing to elevated risk for individuals with pre-existing cardiovascular conditions.

Some associations were negative (shown in Supplementary Materials), implying there is an inverse relationship between increasing levels of air pollution and ED visits for particular strata and pollutants. It is possible that these negative correlations are due to people being aware of reports of elevated $\mathrm{AQHI}$ or other pollutant values for a particular day, and avoid exposing themselves to urban environments where the pollution levels are highest. It is of note that the most negative correlations occur for $\mathrm{NO}_{2}$, seen in Figure e2 (see in Supplementary Materials) for respiratory conditions. The strata/lag combinations with negative associations almost all correspond to strata/lag combinations for $\mathrm{AQHI}$ with null associations (nitrogen dioxide is one of the pollutant factors in the AQHI formula). This could in part explain why there were very few positive $\mathrm{NO}_{2}$ associations in the short-term, even though $\mathrm{NO}_{2}$ has been shown to increase mortality and morbidity rates in this period [28]. While the short-term effect of $\mathrm{NO}_{2}$ on respiratory ED visits was inverse, it was shown that in the longer term (lags 12-14) $\mathrm{NO}_{2}$ was the only mono-pollutant with significant increases in ED visit totals. It is possible that the accumulation of $\mathrm{NO}_{2}$ in the lungs through repeated exposure can lead to delayed respiratory effects, as $\mathrm{NO}_{2}$ is not very water soluble, and therefore does not produce irritation immediately in the upper respiratory tracts but rather deeper into the bronchi $[29,30]$.

To compensate for the high amount of statistical associations being made and thus limit the impact of the multiple comparison problem on inferences made in this paper, a "persistence test" was performed. Index and lag, or strata and lag combinations which had the highest totals of statistically significant positive associations at $p$-value 0.05 in majority remained statistically significant when a more stringent $p$-value $(p<0.001)$ was applied. Visits to the ED in the $0-10$ age group for respiratory problems remained strongly associated to levels of $\mathrm{PM}_{2.5}$ and $\mathrm{SO}_{2}$, as did $\mathrm{O}_{3}$ for almost all strata. For circulatory cases, $\mathrm{NO}_{2}$ remained strongly associated with most strata at lag 0 , while $\mathrm{PM}_{2.5}$ did not.

Given these results and concurrence with available literature, it is reasonable to validate the hypothesis proposed in this study.

The primary limitation of this work is that it does not control for exposure duration, thus it assumes similar exposure for all tracked cases. We also acknowledge that this study does not control for heterogeneity of exposure to air pollution in a population. Individuals may be affected by numerous sources of pollution that may not be registered by an ambient air pollution monitor, such as their proximity to traffic [31-33]. Other sources of exposures which affect human health are cooking emissions [34-36], power plants emissions [37], wildfires [38], and air pollutants generated from active and passive tobacco smoke [39].

Finally, the multiplicity of comparisons performed in this study introduces a possibility of erroneous associations and thus misguided inferences. To combat this, the same analysis using a much more stringent $p$-value than the original $p=0.05$ was contrasted against the latter, and the results showed a persistence of the combinations of strata, lags and pollutants that showed the most statistically significant associations in the primary analysis. 


\section{Conclusions}

This study's objective was to shed light on how fluctuations of air pollution levels affect cardiovascular and respiratory related ED visits with respect to patient age, sex, seasonality, and lag. We have demonstrated that exposure to elevated ambient air pollution levels, particularly $\mathrm{NO}_{2}$ and $\mathrm{O}_{3}$, are positively associated with the number of ED visits related to circulatory and respiratory cases. These correlations were strongest at 0,1 and 3, 4 days post-exposure for circulatory and respiratory illnesses, respectively. It was also reinforced that the common air pollution indicator AQHI is a good multi-pollutant predictor of elevated circulatory and respiratory cases at the specified lags. These conclusions were mostly upheld when using a more stringent $p$-value.

\section{Acknowledgments}

The authors acknowledge Environment Canada for providing the air pollution data from the National Air Pollution Surveillance (NAPS) network.

Parts of this material are based on data and information compiled and provided by the Canadian Institute for Health Information ( $\mathrm{ClHI}$ ). However, the analyses, conclusions, opinions and statements expressed herein are not necessarily those of $\mathrm{CIHI}$.

\section{Additional Materials}

The following additional materials (Supplementary Materials) are uploaded at the location https://github.com/szyszkowiczm/CardioRespirTORONTO. CARDIACRRiskToronto.csv - contains the estimated relative risks for all models (2160) for ED visits for cardiac problems. CARDIACToronto.csv - contains the estimated slopes and their standard errors for all models (2160) for ED visits for cardiac problems. Figure e1 (Cardiac).jpg- a map of the associations for cardiac problems. Figure e2 (Respiratory).jpg - a map of the associations for respiratory problems. Figure e3 (RR, Cardiac, NO2).jpg - forest plot for cardiac and NO2. Figure e4 (RR, Respiratory, O3).jpg forest plot for respiratory and 03. HistAQHI-AQHIX-CO-NO2.jpg - histogram of AQHI, AQHIX, CO, and NO2. HistO3-O3H8-PM25-SO2.jpg - histogram of O3, O3h8, PM2.5, and SO2. HistTempRHum.jpg -histogram of temperature and relative humidity. RESPIRATORYRRiskToronto.csv - contains the estimated relative risks for all models (2160) for ED visits for respiratory problems. RESPIRATORYToronto.csV - contains the estimated slopes and their standard errors for all models (2160) for ED visits for respiratory problems. TorontoMapStation.jpg - map of Toronto: station locations and population density.

\section{Author Contributions}

Mieczysław Szyszkowicz: Conceptualization, Data Curation, Methodology, Software, Supervision, Formal analysis, Writing-original draft, Writing-review \& editing, Project administration; Nicholas de Angelis: Formal analysis, Methodology, Validation, Visualization; Writing-review \& editing.

\section{Competing Interests}

The authors have declared that no competing interests exist. 


\section{References}

1. Forero R, McCarthy S, Hillman K. Access block and emergency department overcrowding. Crit Care. 2011; 15: 216.

2. Hoot NR, Aronsky D. Systematic review of emergency department crowding: Causes, effects, and solutions. Ann Emerg Med. 2008; 52: 126-136.

3. Sprivulis PC, Da Silva JA, Jacobs IG, Frazer AR, Jelinek GA. The association between hospital overcrowding and mortality among patients admitted via Western Australian emergency departments. Med J Aust. 2006; 184: 208-212.

4. CIHI. NACRS emergency department visits and length of stay by province/territory, 2018-2019. Ottawa, ON: ClHI; 2019.

5. Burnett R, Chen H, Szyszkowicz M, Fann N, Hubbel B, Pope CA, et al. Global estimates of mortality associated with long-term exposure to outdoor fine particulate matter. Proc Natl Acad Sci USA. 2018; 115: 9592-9597.

6. Hoffmann B, Moebus S, Möhlenkamp S. Residential exposure to traffic is associated with coronary atherosclerosis. Circulation. 2007; 116: 489-496.

7. Künzli N, Jerrett M, Mack WJ, Beckerman B, LaBree L, GillilandF, et al. Ambient air pollution and atherosclerosis in Los Angeles. Environ Health Persp. 2005; 113: 201-206.

8. Peters A, Klot S, Mittleman MA. Triggering of acute myocardial infarction by different means of transportation. Eur J Prev Cardiol. 2013; 20: 750-758.

9. Miller MR, Newby DE. Air pollution and cardiovascular disease: Car sick. Cardiovasc Res. 2020; 116: 279-294.

10. Kim D, Chen Z, Zhou LF, Huang SX. Air pollutants and early origins of respiratory diseases. Chronic Dis Transl Med. 2018; 4: 75-94.

11. Vicedo-Cabrera AM, Sera F, Liu C, Armstrong B, Milojevic A, Guo Y, et al. Short term association between ozone and mortality: global two stage time series study in 406 locations in 20 countries. BMJ. 2020; 368: m108.

12. Stieb DM, Burnett RT, Smith-Doiron M, Brion O, Shin HH, Economou V. A new multipollutant, no-threshold air quality health index based on short-term associations observed in daily timeseries analyses. J Air Waste Manag Assoc. 2008; 58: 435-450.

13. Szyszkowicz M. Use of generalized linear mixed models to examine the association between air pollution and health outcomes. Int J Occup Med Environ Health. 2006; 19: 224-227.

14. Armstrong BG, Gasparrini A, Tobias A. Conditional Poisson models: A flexible alternative to conditional logistic case cross-over analysis. BMC Med Res Methodol. 2014; 14: 122.

15. Szyszkowicz M. Case-crossover method with a short time-window. Int J Environ Res Public Health. 2019; 17: 202.

16. Szyszkowicz M. Use of two-point models in "model choice in time-series studies of air pollution and mortality". Air Qual Atmos Health. 2020; 13: 225-232.

17. D'Amato M, Molino A, Calabrese G, Cecchi L, Annesi-Maesano I, D'Amato G. The impact of cold on the respiratory tract and its consequences to respiratory health. Clin Transl Allergy. 2018; 8: 20.

18. Mokoena KK, Ethan CJ, Yu Y, Shale K, Liu F. Ambient air pollution and respiratory mortality in Xi'an, China: A time-series analysis. Respir Res. 2019; 20: 139. 
19. Stieb DM, Szyszkowicz M, Rowe BH, Leech JA. Air pollution and emergency department visits for cardiac and respiratory conditions: A multi-city time-series analysis. Environ Health. 2009; 8: 25.

20. Ho A, Zheng $H$, Earnest A. Time-stratified case crossover study of the association of outdoor ambient air pollution with the risk of acute myocardial infarction in the context of seasonal exposure to the southeast Asian haze problem. J Am Heart Assoc. 2019; 8: e011272.

21. Kurt OK, Zhang J, Pinkerton KE. Pulmonary health effects of air pollution. Curr Opin Pulm Med. 2016; 22: 138-143.

22. Rodríguez-Villamizar LA, Rojas-Roa NY, Blanco-Becerra LC, Herrera-Galindo VM, FernándezNiño JA. Short-term effects of air pollution on respiratory and circulatory morbidity in Colombia 2011-2014: A multi-city, time-series analysis. Int J Environ Res Public Health. 2018; 15: 1610.

23. Alhanti BA, Chang HH, Winquist A, Mulholland JA, Darrow LA, Sarnat SE. Ambient air pollution and emergency department visits for asthma: A multi-city assessment of effect modification by age. J Expo Sci Environ Epidemiol. 2016; 26: 180-188.

24. Ohlwein S, Kappeler R, Kutlar Joss M, Künzli N, Hoffmann B. Health effects of ultrafine particles: A systematic literature review update of epidemiological evidence. Int J Public Health. 2019; 64: 547-559.

25. Luo K, Li R, Li W, Wang Z, Ma X, Zhang R, et al. Acute effects of nitrogen dioxide on cardiovascular mortality in Beijing: An exploration of spatial heterogeneity and the districtspecific predictors. Sci Rep. 2016; 6: 38328.

26. Wu CF, Shen FH, Li YR, Tsao TM, Tsai MJ, Chen CC, et al. Association of short-term exposure to fine particulate matter and nitrogen dioxide with acute cardiovascular effects. Sci Total Environ. 2016; 569-570: 300-305.

27. Fares A. Winter cardiovascular diseases phenomenon. N Am J Med Sci. 2013; 5: 266-279.

28. Samoli E, Aga E, Touloumi G, Anderson HR, Schindler C, Forsberg B, et al. Short-term effects of nitrogen dioxide on mortality: An analysis within the APHEA project. Eur Respir J. 2006; 27: 1129-1138.

29. Gorguner M, Akgun M. Acute inhalation injury. Eurasian J Med. 2010; 42: 28-35.

30. Schraufnagel DE, Balmes JR, Cowl CT, Matteis SD, Jung SH, Matteis K, et al. Air Pollution and noncommunicable diseases: A review by the forum of international respiratory societies' environmental committee, part 2: Air pollution and organ systems. Chest. 2019; 155: 417-426.

31. Özkaynak H, Baxter LK, Dionisio KL, Burke J. Air pollution exposure prediction approaches used in air pollution epidemiology studies. J Expo Sci Environ Epidemiol. 2013; 23: 566-572.

32. Szyszkowicz M, Angelis N. Ambient air pollution and emergency department visits in Toronto, Canada. Environ Sci Pollut Res Int. 2021; 28: 28789-28796.

33. Szyszkowicz M, Schoen S, Angelis N. Air pollution and emergency department visits for disease of the genitourinary system. Environ Health Insights. 2021. doi: 10.1177/11786302211025360.

34. Zhang Q, Gangupomu RH, Ramirez D, Zhu Y. Measurement of ultrafine particles and other air pollutants emitted by cooking activities. Int J Environ Res Public Health. 2010; 7: 1744-1759.

35. Robinson ES, Gu P, Ye Q, Li HZ, Shah RU, Apte JS, et al. Restaurant impacts on outdoor air quality: Elevated organic aerosol mass from restaurant cooking with neighborhood-scale plume extents. Environ Sci Technol. 2018; 52: 9285-9294. 
36. Shah RU, Robinson ES, Gu P, Apte JS, Marshall JD, Robinson AL, et al. Socio-economic disparities in expo-sure to urban restaurant emissions are larger than for traffic. Environ Res Lett. 2020; 15. doi: 10.1088/1748-9326/abbc92.

37. Daouda M, Henneman L, Kioumourtzoglou MA, Gemmill A, Zigler C, Casey JA. Association between county-level coal-fired power plant pollution and racial disparities in preterm births from 2000 to 2018. Environ Res Lett. 2021; 6. doi: 10.1088/1748-9326/abe4f7.

38. Reid CE, Brauer M, Johnston FH, Jerrett M, Balmes JR, Elliott CT. Critical review of health impacts of wildfire smoke exposure. Environ Health Persp. 2016; 124: 1334-1343.

39. Georgiadis P, Stoikidou M, Topinka J, Kaila S, Gioka M. Personal exposures to PM2.5 and polycyclic aromatic hydrocarbons and their relationship to environmental tobacco smoke at two locations in Greece. J Expo Anal Environ Epid. 2021; 11: 169-183.

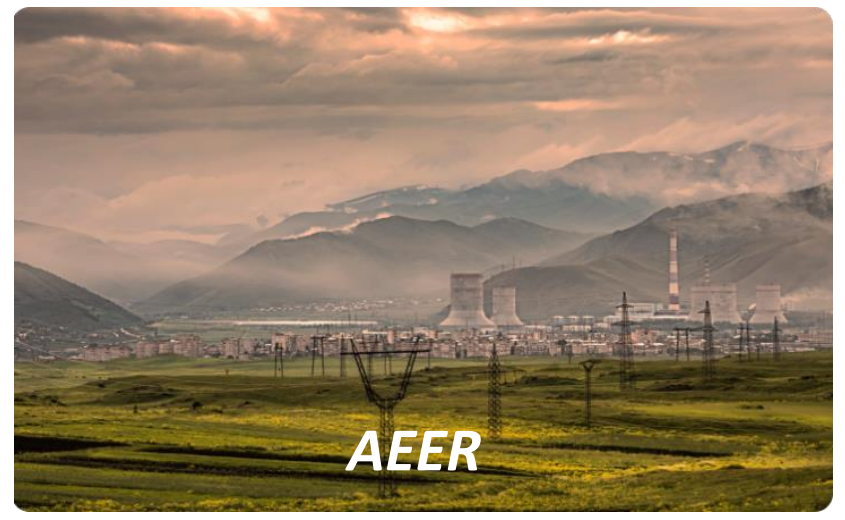

Enjoy AEER by:

1. Submitting a manuscript

2. Joining in volunteer reviewer bank

3. Joining Editorial Board

4. Guest editing a special issue

For more details, please visit:

http://www.lidsen.com/journals/aeer 